\title{
Late Onset of Wolff-Parkinson-White Syndrome in a 72-year-old Man
}

\author{
Masao TAKemoto, Hideki Origuchi, Junji Kawagoe, Nobuya Harayama, \\ Yasuhide SodA*, Hideo Yamamoto and Hitoshi YoshimuRA
}

\begin{abstract}
We encountered a case of wide QRS tachycardia with chronic atrial fibrillation in Wolff-Parkinson-White syndrome. Unique features were late onset of syncope attacks associated with this tachycardia at an advanced age of 72 years old without previous documentation of WolffParkinson-White syndrome on electrocardiogram. He had a high likelihood of sudden cardiac death. Catheter ablation using CARTO ${ }^{\mathrm{TM}}$ system easily led to a successful ablation of the accessory pathway. The mechanism of late onset of the wide QRS tachycardia was attributed to possible changes of electrophysiologic properties including the atrio-ventricular node and/or the accessory pathway, and the unique location of the accessory pathway.
\end{abstract}

(Internal Medicine 43: 939-944, 2004)

Key words: wide QRS complex tachycardia, syncope, Wolff-Parkinson-White syndrome, atrial fibrillation, catheter ablation, aging

\section{Case Report}

A 72-year-old man was admitted for evaluation of frequent syncope or fainting attacks which had suddenly begun 1 month before admission in May 2002. He easily experienced syncope attacks on exercise. Thus, his daily life activities were limited. He was taking antihypertensive drugs (5 $\mathrm{mg}$ of enalapril and $25 \mathrm{mg}$ of atenolol), digitalis glycosides (0.125 $\mathrm{mg}$ of digoxin) and warfarin potassium as anticoagulant therapy for $\sim 10$ years because of a history of cerebral infarction associated with hypertension and non-valvular chronic atrial fibrillation (Af). An electrocardiogram (ECG) taken 1 year prior to the admission was Af with narrow QRS complex (Fig. 1A) with no evidence of tachycardia and delta waves (mean heart rate; 64 beats per minute, QRS; $82 \mathrm{msec}$ ). While the ECG taken 2 months prior to the admission showed Af with intermittent delta waves (mean heart rate; 60 beats per minute) (Fig. 2A).

On admission, his blood pressure was 102/64 $\mathrm{mmHg}$ and pulse rate was 124 beats per minute and irregular. When his heart rate was over 150 beats per minute on exercise, his systolic blood pressure dropped under $70 \mathrm{mmHg}$ with manifestation of fainting. There were no murmurs on cardiac auscultation. Routine blood test results were almost normal except for a coagulation time. An ECG (Fig. 1B) showed wide QRS complex tachycardia (mean heart rate; 138 beats per minute, QRS; $134 \mathrm{msec}$ ) with a slur at the initial portion of the QRS complex. This tachycardia was suppressed by an intravenous administration of procainamide, which is known to depress the conduction of the accessory pathway (1), and resulted in a dramatic decrease of the wide QRS complex (Fig. 2B). By contrast, the tachycardia was accelerated by an intravenous application of verapamil or digitalis glycosides (Fig. 2C), which are reported to depress the conduction of the atrio-ventricular (AV) node $(2,3)$. With this change of ECG, the patient experienced symptoms of hypotension and notable fainting. These findings suggested that this patient might have Wolff-Parkinson-White (WPW) syndrome. A chest radiograph showed a mild increase of the cardiothoracic ratio (CTR; 54\%) and two-dimensional echocardiography revealed no evidence of cardiac abnormalities except for mild dilatation of the left atrium, such as Ebstein's anomaly and mitral leaflet prolapse which are reported to often associate with WPW syndrome $(4,5)$. The QRS and delta wave morphologies were positive in $\mathrm{V}_{1}$ and negative in II, III, and $\mathrm{aV}_{\mathrm{F}}$ (Fig. 1B), indicating that the accessory pathway was located in the posteroseptal position of the left ventricle (6). His shortest R-R interval was measured to be 248 msec (Fig. 2D), which was compatible with the criteria for high risk of the development of ventricular fibrillation and sudden death in patients with WPW syndrome (7). Since oral

From the Internal Medicine, Kyushu Kosei-Nenkin Hospital, Kitakyushu and *the Internal Medicine, Soda Hospital, Nobeoka

Received for publication November 28, 2003; Accepted for publication May 31, 2004

Reprint requests should be addressed to Dr. Masao Takemoto, the Internal Medicine, Kyushu Kosei-Nenkin Hospital, 1-8-1 Kishinoura, Yahatanishi-ku, Kitakyushu 806-8501 
A

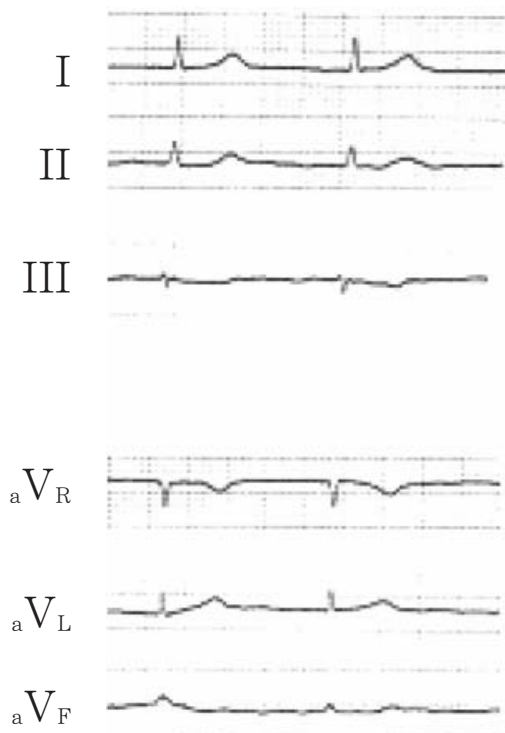

$\mathrm{V}_{1}$
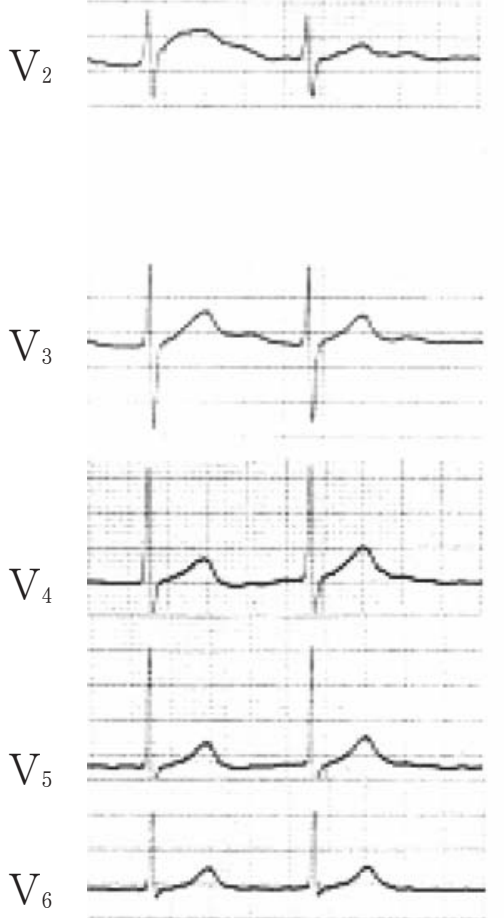

B

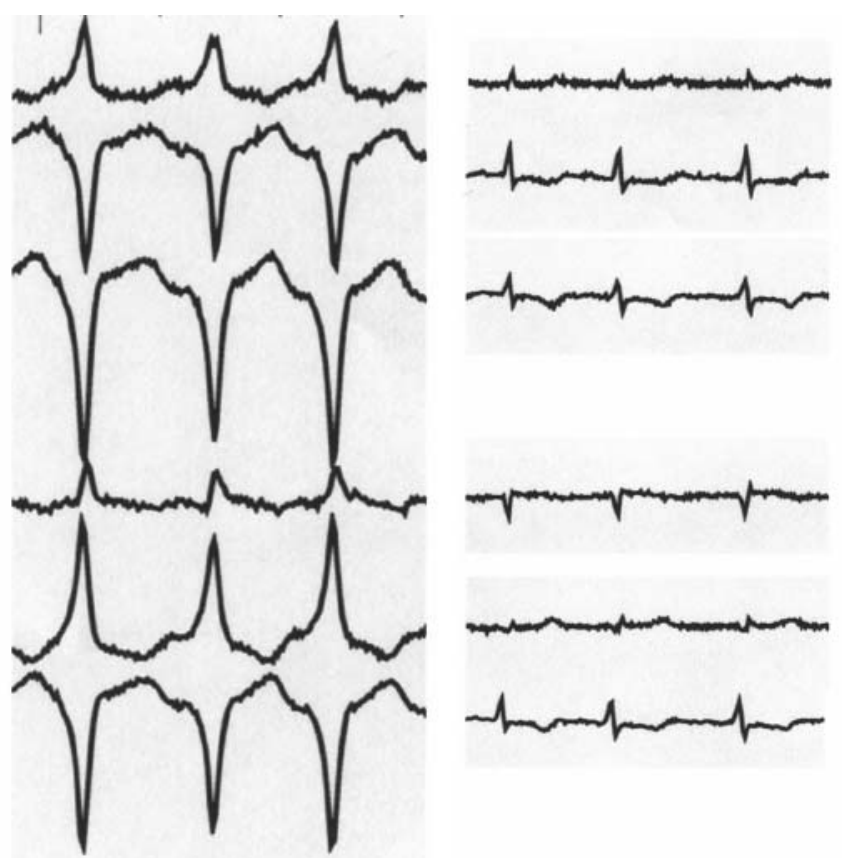

\section{(1)}
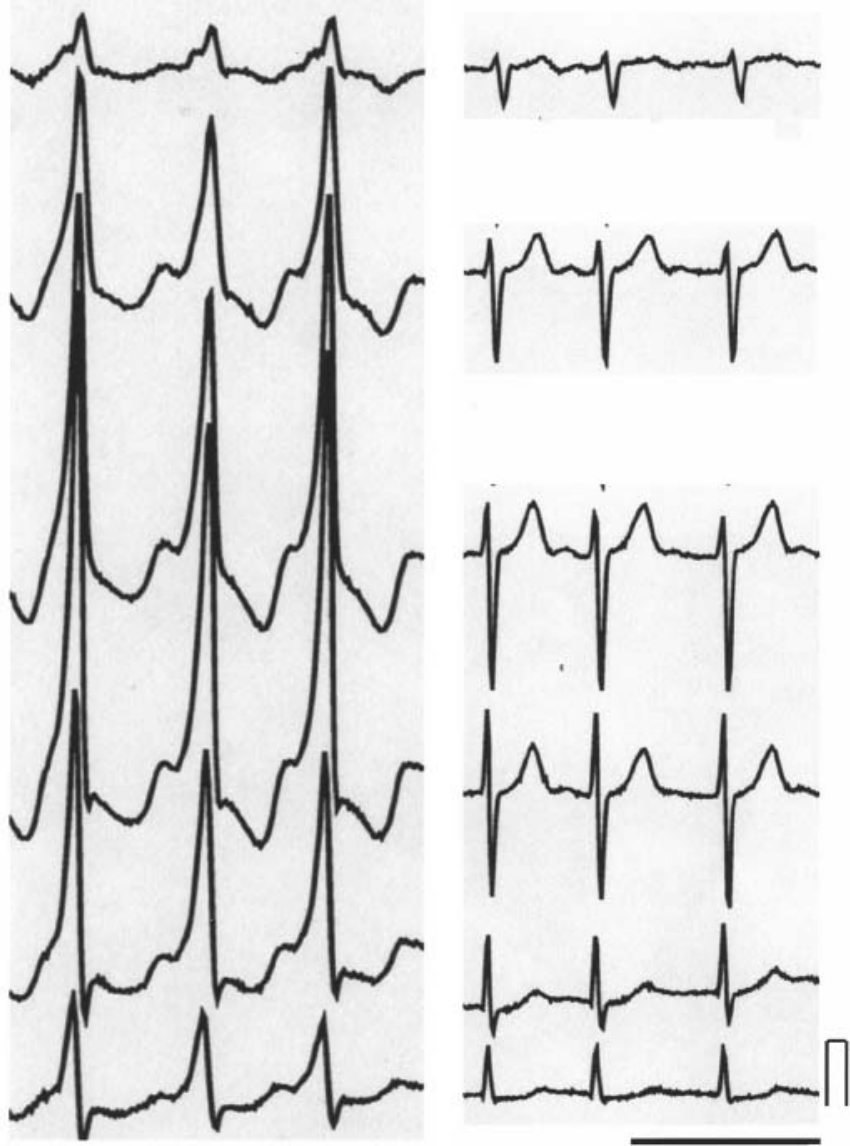

Figure 1. The ECG taken 1 year prior to admission (A) showed Af with narrow QRS complex without tachycardia (mean heart rate; 64 beats per minute, QRS duration; $82 \mathrm{msec}$ ). On the other hand, the ECG on admission (B) showed wide QRS tachycardia (mean heart rate; 138 beats per minute, QRS duration; $134 \mathrm{msec}$ ). After ablation $(\mathrm{C})$, the wide QRS tachycardia could not be induced even after an intravenous administration of isoproterenol (0.2 $\mu \mathrm{g}$ per minute). Bar indicates 1,000 msec. 
A

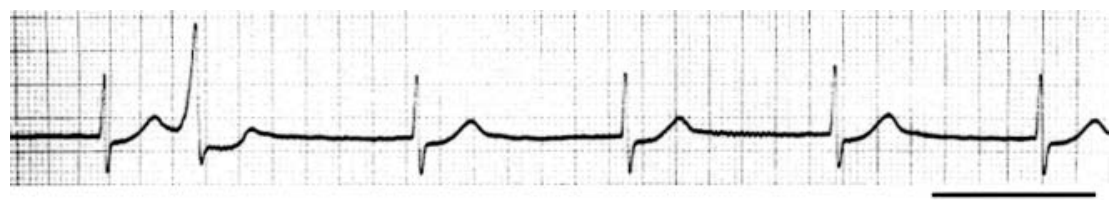

B

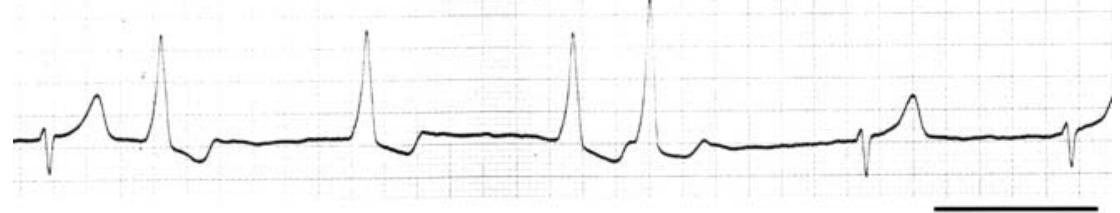

C

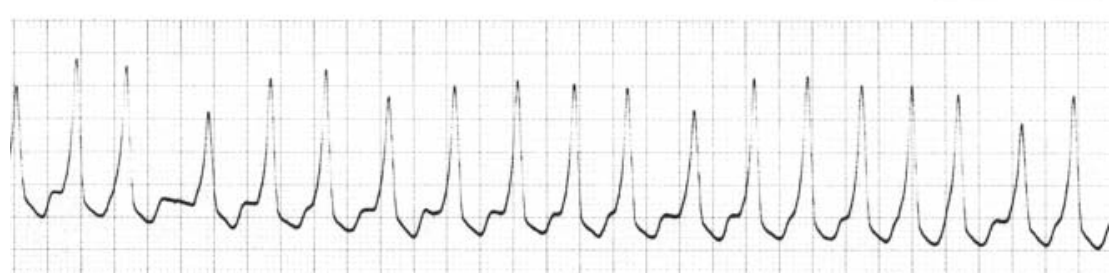

D

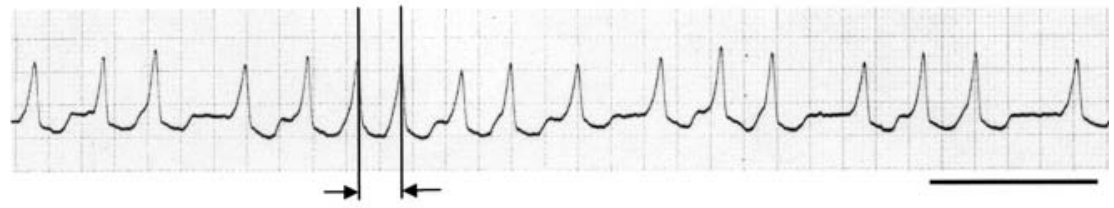

SRR interval

$E$

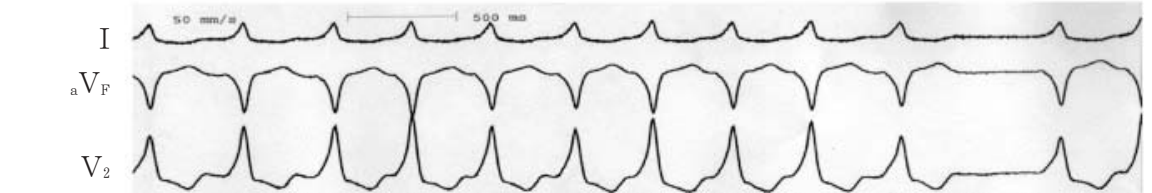

His 1024 CS 10 (1)

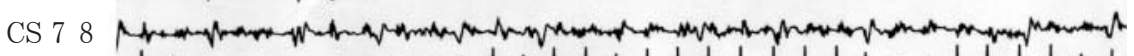

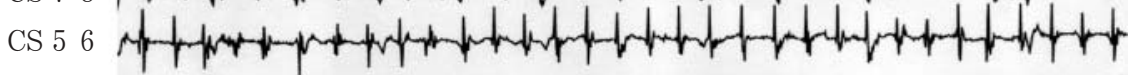

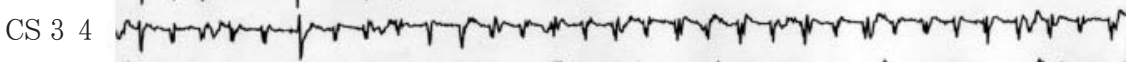
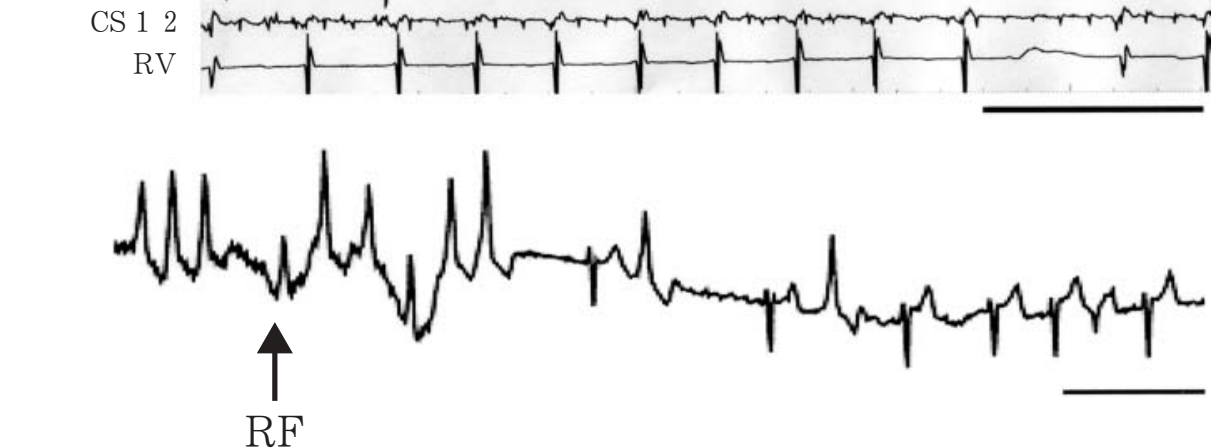

Figure 2. The ECG taken 2 months prior to admission showed Af with intermittent delta waves (A). The wide QRS tachycardia was suppressed by procainamide (mean heart rate; 63 beats per minute) with a partial disappearance of wide QRS complex (B), and accelerated by verapamil or digitalis glycosides (mean heart rate; 172 beats per minute)(C). The shortest R-R (SRR) interval was 248 msec during wide QRS tachycardia (D). The endocardial electrogram demonstrated irregular activities with altered amplitude, findings compatible with Af (E). After delivering the radio-frequency ( $R F$ ) energy, the wide QRS tachycardia with the delta wave readily terminated (F). Bar indicates $\mathbf{1 , 0 0 0}$ msec. 
administration of class IA, IC, or III agents failed to terminate this wide QRS tachycardia, the electrophysiological studies were performed prior to the proposed catheter ablation, after receiving informed consent. The electrical defibrillation was not considered because of the presence of thrombi in the left atrial appendage confirmed by transesophageal echocardiography.

The 4-French deflectable catheters were inserted into the right ventricle, His-bundle, and coronary sinus. The endocardial electrogram demonstrated irregular activities with altered amplitude, findings compatible with Af (Fig. 2E). The analysis of electroanatomical cardiac mapping using CARTO $^{\mathrm{TM}}$ system (8) (Biosense Webster Ltd., TriantHaCarmel, Israel) was performed to determine the earliest site of the rapid ventricular response ( $\mathrm{V}$ wave) over the accessory pathway. The locatable mapping catheter was introduced under fluoroscopic guidance and was positioned inside the heart chambers to be mapped. Both activation and propagation maps produced by CARTO ${ }^{\mathrm{TM}}$ system revealed that the earliest site of the $\mathrm{V}$ wave was at the posterior septum in the left ventricle (Fig. 3A and B). The ablation catheter was introduced under the guidance of this map to the target site which showed a QS-pattern when examined by the endocardial electrogram recorded by the tip of the ablation catheter. Immediately after delivering the radio-frequency energy to this site, the wide QRS tachycardia with the delta wave was readily terminated (Fig. 2F). After the ablation, the electrophysiological studies showed normal HV conduction time $(35 \mathrm{msec})$. On the other hand, the effective refractory period (ERP) of AV node could not be determined because of the sustained presence of Af. The wide QRS tachycardia was unable to be induced even by an intravenous administration of isoproterenol ( $0.2 \mu \mathrm{g}$ per minute) (Fig. 1C).

He never complained of syncope or fainting after the catheter ablation, and has remained well without any symptoms, when last seen in April 2004.

\section{Discussion}

WPW syndrome is characterized by the presence of preexcitation on the ECG and the occurrence of paroxysmal tachycardia. Although the accessory pathway is congenital, its manifestations can be occasionally detected in adolescence and adulthood $(9,10)$. We experienced a case of wide QRS tachycardia with chronic Af in a patient with WPW syndrome. Chronic Af is rare in WPW syndrome (11), whereas the propensity for Af is well shown to be higher in older patients (10). A unique and rare feature of this case was the recent onset of syncope attacks associated with this tachycardia, evolving at a fairly advanced age of 72 years old, without previous documentation of WPW syndrome in ECG. The prevalence of tachyarrhythmias has been reported to decrease with age in WPW syndrome $(9,12,13)$. Although its mechanism is uncertain, some degenerative changes of the accessory pathways developing with age and ensuring prolongation of the ERP have been proposed (10,
14, 15). The manifestation of tachyarrhythmia during Af in WPW syndrome should be a wide QRS tachycardia, when the impulses from the atria that propagate across the accessory pathway capture most of the ventricle. This might have actually occurred in this patient in which either orthodromic or antidromic AV reciprocating tachycardia seems difficult to maintaine due to rapid atrial rates during Af.

The present patient had a sudden onset of symptomatic WPW syndrome with wide QRS tachycardia that occurred 1 month prior to admission. The presumed mechanism of late onset of WPW syndrome is the decrease in the capability of AV node conduction which may progress with age, resulting in total activation of the ventricle through the anterograde conduction over the accessory pathway. A previous study has shown that the capability of AV node conduction is decreased in older patients with WPW syndrome (10). In keeping with the observation, the mean heart rate of the present patient decreased gradually from 74 to 64 beats per minute although he was kept under practically the same medication for about the previous 5 years, and he had 60 beats per minute after the ablation. These findings suggest that impulse conduction through the AV node gradually deteriorated with age and that chronic oral administration of beta-adrenergic receptor antagonist and digitalis glycosides might have further impaired the AV node conduction, relatively enhanced the impulse propagation via the accessory pathway and increased ventricular response.

Another contributing factor may be the site of the accessory pathway. Provided that the site was relatively far from the sinus node, for example, a left lateral accessory pathway, most of the ventricle could be activated by the impulse conducted through the AV node even though the its conduction was somewhat impaired. In the present case, images by CARTO $^{\mathrm{TM}}$ system demonstrated the accessory pathway located at the posterior septum in the left ventricle (Fig. 3A and B) which was relatively close to the sinus node. Thus, the ventricle may be activated mostly by conduction through the accessory pathway, as was the case in this patient.

For most patients with recurrent tachycardia in WPW syndrome, the prognosis is reported to be fairly good, albeit sudden cardiac death occurs with an estimated rate of about 0.1 $\%(16,17)$. In the present case, the shortest R-R interval was $248 \mathrm{msec}$ (Fig. 2D) and the preexitation was accelerated during exercise; these findings are in agreement with the criteria for high risk of the development of ventricular fibrillation and sudden death in patients with WPW syndrome (7, 18, 19). Moreover, this wide QRS tachycardia was refractory to antiarrhythmic drugs. In such a patient, we successfully ablated the accessory pathway using CARTO ${ }^{\mathrm{TM}}$ system and terminated the occurrence of ventricular tachycardia and associated syncope. These findings may add some merit for using this system in patients of WPW syndrome with chronic Af and recurrent ventricular tachycardias produced by a frequent antegrade propagation of atrial impulses through the accessory pathway.

In summary, we encountered a unique case of late onset 

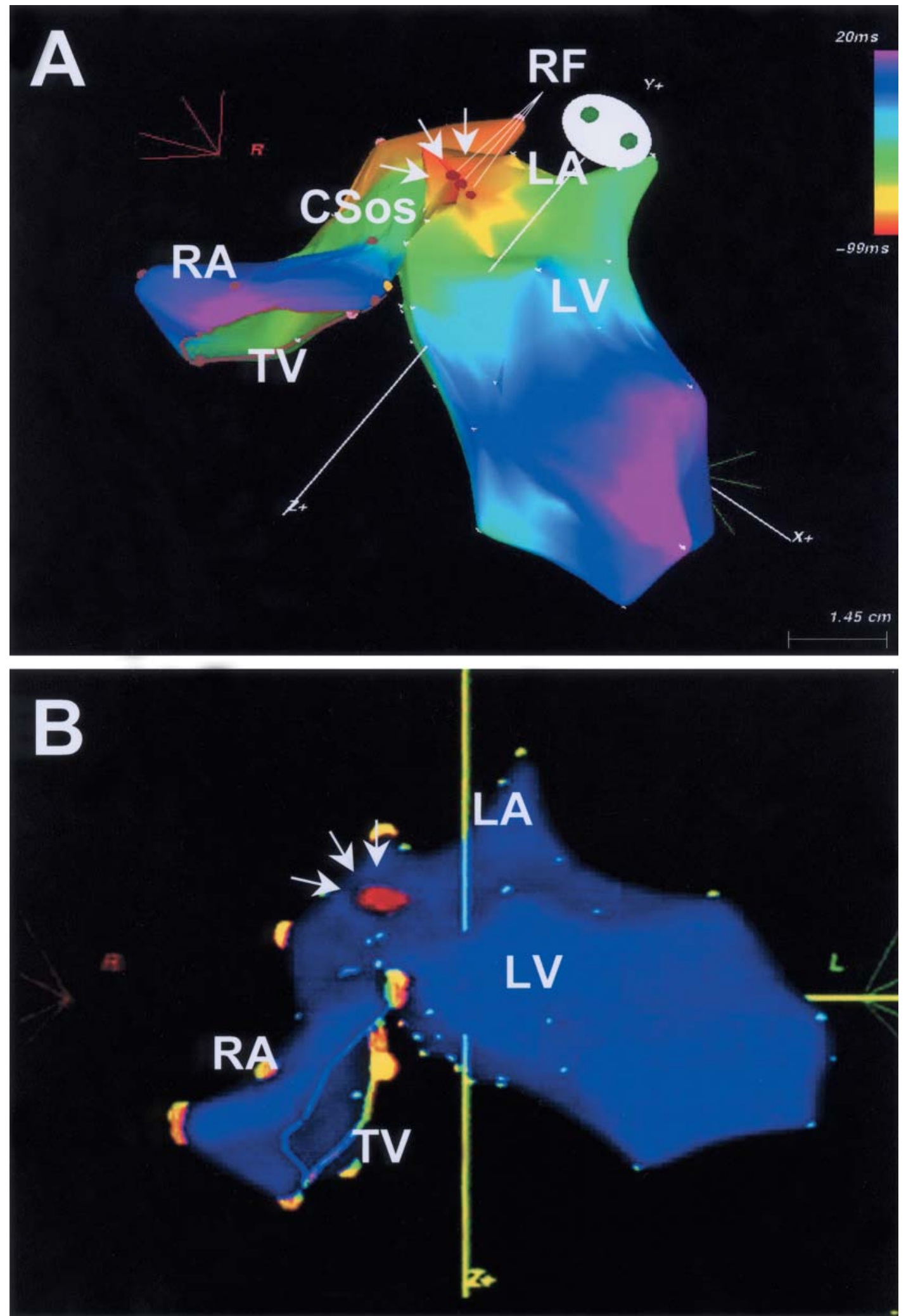

Figure 3. The electroanatomical cardiac mapping showed the presence of the accessory pathway on the left side and the earliest site of the $V$ wave was at the posterior septum in the left ventricle colored orange (arrows) in the activation map (A; LAO view) and colored red (arrows) in the propagation map (B; RAO view). RF indicates ablation sites; RA: right atrium, LA: left atrium, LV: left ventricle, TV: tricuspid valve annulus, CSos: coronary sinus ostium. 


\section{TAKEMOTO et al}

of WPW syndrome at an advanced age without previous documentation of tachyarrhythmias and delta waves. The mechanism of late onset of the wide QRS tachycardia has not been completely elucidated; the changes in electrophysiologic variables including the abilities of the conduction of AV node and/or the accessory pathway which are affected by antiarrhythmic agents, aging, or other factors, and the location of the accessory pathway may be important.

Acknowledgements: The authors thank for Fumiko Hori, Yukimi Takahashi, Hiroko Ohnagamitsu, and Kenji Sakuma for their technical assistance of electrophysiological study and catheter ablation. The authors are also deeply indebted to Makoto Arita, MD for his useful suggestions.

\section{References}

1) Sellers TD Jr, Campbell RW, Bashore TM, Gallagher JJ. Effects of procainamide and quinidine sulfate in the Wolff-Parkinson-White syndrome. Circulation 55: 15-22, 1977.

2) Gulamhusein S, Ko P, Klein GJ. Ventricular fibrillation following verapamil in the Wolff-Parkinson-White syndrome. Am Heart J 106: 145-147, 1983

3) Sellers TD Jr, Bashore TM, Gallagher JJ. Digitalis in the pre-excitation syndrome. Analysis during atrial fibrillation. Circulation 56: 260-267, 1977.

4) Pressley JC, Wharton JM, Tang AS, Lowe JE, Gallagher JJ, Prystowsky EN. Effect of Ebstein's anomaly on short- and long-term outcome of surgically treated patients with Wolff-Parkinson-White syndrome. Circulation 86: 1147-1155, 1992.

5) Rechavia E, Mager A, Birnbaum Y, Sclarovsky S. Mitral valve prolapse, sick sinus and Wolff-Parkinson-White syndromes: interrelationships with respect to sudden cardiac death. Isr J Med Sci 29: 654 655, 1993.

6) Xie B, Heald SC, Bashir Y, et al. Localization of accessory pathways from the 12-lead electrocardiogram using a new algorithm. Am J Cardiol 74: 161-165, 1994.
7) Klein GJ, Bashore TM, Sellers TD, Pritchett EL, Smith WM, Gallagher JJ. Ventricular fibrillation in the Wolff-Parkinson-White syndrome. N Engl J Med 301: 1080-1085, 1979.

8) Drago F, Silvetti MS, Di Pino A, Grutter G, Bevilacqua M, Leibovich S. Exclusion of fluoroscopy during ablation treatment of right accessory pathway in children. J Cardiovasc Electrophysiol 13: 778-782, 2002.

9) Braunwald E. Specific Arrhythmias: Diagnosis and Treatment. Heart Disease. 5th. ed. Saunders, Philadelphia, 1997: 640-704.

10) Fan W, Peter CT, Gang ES, Mandel W. Age-related changes in the clinical and electrophysiologic characteristics of patients with WolffParkinson-White syndrome: comparative study between young and elderly patients. Am Heart J 122: 741-747, 1991.

11) Zipes D, Jalife J. The Wolff-Parkinson-White Syndrome and Related Variants. Cardiac Electrophysiology: From Cell to Bedside. 3rd. ed. Saunders, Philadelphia, 2000: 845-861.

12) Giardina AC, Ehlers KH, Engle MA. Wolff-Parkinson-White syndrome in infants and children. A long-term follow-up study. Br Heart J 34: 839-846, 1972.

13) Perry JC, Garson A Jr. Supraventricular tachycardia due to WolffParkinson-White syndrome in children: early disappearance and late recurrence. J Am Coll Cardiol 16: 1215-1220, 1990.

14) Klein GJ, Hackel DB, Gallagher JJ. Anatomic substrate of impaired antegrade conduction over an accessory atrioventricular pathway in the Wolff-Parkinson-White syndrome. Circulation 61: 1249-1256, 1980.

15) Michelucci A, Padeletti L, Fradella GA, et al. Aging and atrial electrophysiologic properties in man. Int J Cardiol 5: 75-81, 1984.

16) Zardini M, Yee R, Thakur RK, Klein GJ. Risk of sudden arrhythmic death in the Wolff-Parkinson-White syndrome: current perspectives. Pacing Clin Electrophysiol 17: 966-975, 1994.

17) Munger TM, Packer DL, Hammill SC, et al. A population study of the natural history of Wolff-Parkinson-White syndrome in Olmsted County, Minnesota, 1953-1989. Circulation 87: 866-873, 1993.

18) Pappone C, Santinelli V, Manguso F, et al. A randomized study of prophylactic catheter ablation in asymptomatic patients with the WolffParkinson-White syndrome. N Engl J Med 349: 1803-1811, 2003.

19) Lerman BB, Basson CT. High-risk patients with ventricular preexcitation—a pendulum in motion. N Engl J Med 349: 1787-1789, 2003. 